\title{
Survey on Data Deduplication of Text File over Cloud
}

\author{
Walzade Arti K. ${ }^{1}$, Zine Datta ${ }^{2}$ \\ ${ }^{1}$ Savitribai Phule Pune University, Vishwabharati Academics COE, Ahmednagar \\ ${ }^{2}$ Professor, Savitribai Phule Pune University, Vishwabharati Academics COE, Ahmednagar
}

\begin{abstract}
Now a days cloud technology are being continuously used in IT field as well as in other to keep the data, cloud re-copying is also such service which has to be focused on. As the cloud service has improved huge focus in last few year. Cloud storage massive management has become important. This document surveying various works previously done in the area of cloud services and therefore, re-copying of data over cloud storages has still scope of improvement. Inspection on the papers or researches emphasizes various deduplication idea and the ways they are differ from each other for efficient deduplication. Thus as there are many ways of deduplication in clouds, an efficient technique is to be search out having less drawbacks and more outcome.
\end{abstract}

Keywords: re-copying, POW, hybrid storage on cloud, open clouds, credentials, robustly.

\section{Introduction}

Current time is distributed computing time. Distributed computing has massive variety of degree in data sharing in current period. Distributed computing is give accurate measure of virtual environment concealing the stage and working frameworks of the client. Client use the assets for exchanging information. It may, client need to pay by the process of utilization of assets of cloud. Now cloud admin distributors are putting forth cloud administrations with ease furthermore with large dependability. Client can transfer the vast sum data on cloud and exchanged information to a large number of clients. Cloud suppliers are offer diverse administrations, for example, framework as an administration, stage as an administration, and so forth. Clients not have to buy the assets. As the data is get exchanged by the client might be it is basic notification to deal with this regularly expanding information on the cloud. To make well information administration in the distributed computing. We use duplication technique, which is the best technique in cloud. This technique is turning out to be more moderation for information DE duplication. This system is sending the information over the system required little measure of information. This technique has application in information administration and organizing. Information duplication is the procedure of decreasing copy file Also it is the best pressure system for the information DE duplication. This system have application in information administration and organizing. Rather than keeping excess duplicate file of the same information DE duplication just keep unique duplicate and give just references of the first duplicate to the repetitive information. The processes of checking the duplication process are two, one is document level duplication check and other is piece content level duplication check. In the document level duplication technique check is expel the same name record from the capacity and square level DE duplication are evacuated the copy pieces. DE duplication techniques need of the some security system. In the conventional system client need to encode his own particular information.

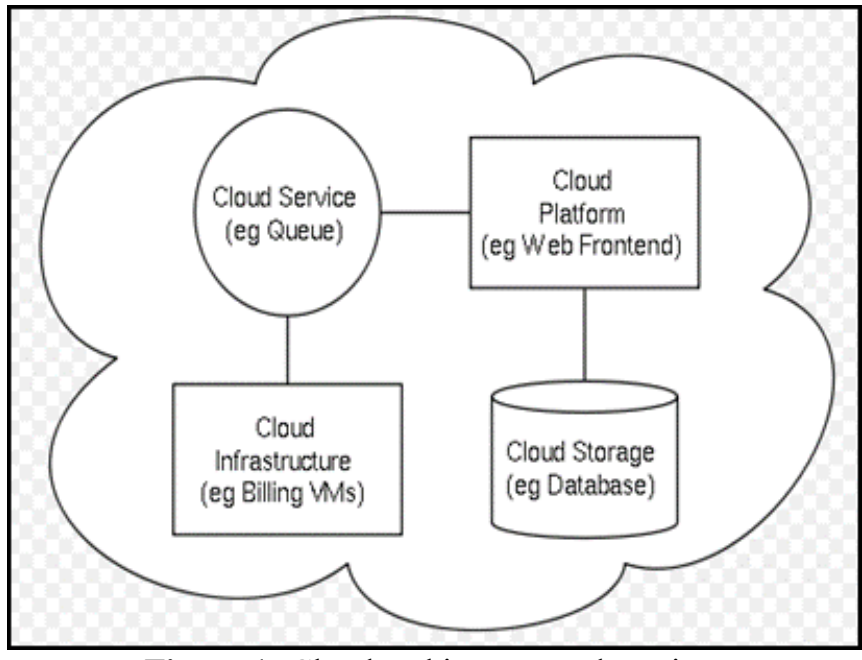

Figure 1: Cloud architecture and services

To maintain a security from the unapproved information DE duplication focalized information DE duplication is proposed to uphold the data privacy while checking the information duplication. The cloud giving various administrations as attended in the above figure, for example, stage, administrations, base as an administration, and database as an administration.

In this we are utilizing as a part of distributed storage as an administration.

We are utilizing client accreditations to check the confirmation of the client. In that cases cloud is available two sort of cloud such private cloud and open cloud. In private cloud store the client accreditation and in the open cloud client information present out. In the figure 2 cloud take focal points of both open cloud and private cloud. open cloud and private cloud are available in the half and half cloud structural engineering. When any client forward solicitation to people in general cloud to get to the data he have to present his data to the private cloud then private cloud will give a record token and client can get the notifications to the document lives on the general population cloud. We have utilized a half and half cloud construction modeling as a part of proposed. We have to need to mind the file name in record

Volume 6 Issue 1, January 2017 


\section{International Journal of Science and Research (IJSR) \\ ISSN (Online): 2319-7064 \\ Index Copernicus Value (2015): 78.96 | Impact Factor (2015): 6.391}

information duplication and information DE duplication is checked at the square level. On the other hand, client needs to recover his information or download the information record he have to download both of the document from the cloud server this will prompts perform the operation on the same record this abuses the security of the distributed storage.

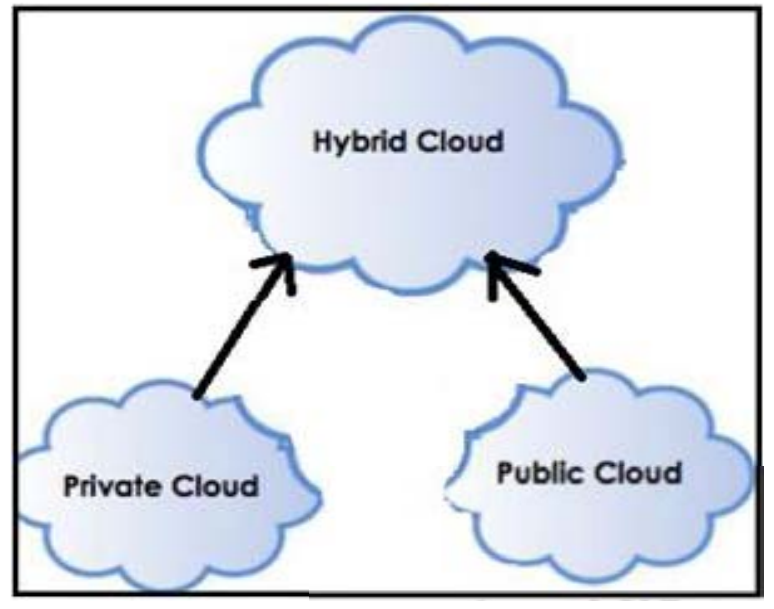

Figure 2: Hybrid Cloud Architecture

\section{Literature Survey}

\section{A. DupLESS: Server-Aided Encryption for Deduplicated Storage}

By looking the example Dropbox, Mozy, and others perform deduplication to spare space by just putting away one duplicate of every document transferred. Should customers routinely scramble their documents, be that as it may, funds are lost. Message-bolted encryption (the most unmistakable appearance of which is concurrent encryption) determines this strain. In any case it is intrinsically subject to savage power assaults that can recoup records falling into a known set. We propose a building design that acevides secure deduplicated stockpiling opposing savage power assaults, and acknowledge it in a framework called DupLESS. In DupLESS, customers encode under message-based keys acquired from a key-server by means of an absent PRF convention. It securies customers to store scrambled information with a current administration, have the administration perform deduplication for their benefit, but then accomplishes solid privacy ensures. We demonstrate that encryption for deduplicated stockpiling can accomplish execution and space reserve funds near that of utilizing the stockpiling administration with plaintext information [1].

\section{B. Fast and Secure Laptop Backups with Encrypted De-} duplication

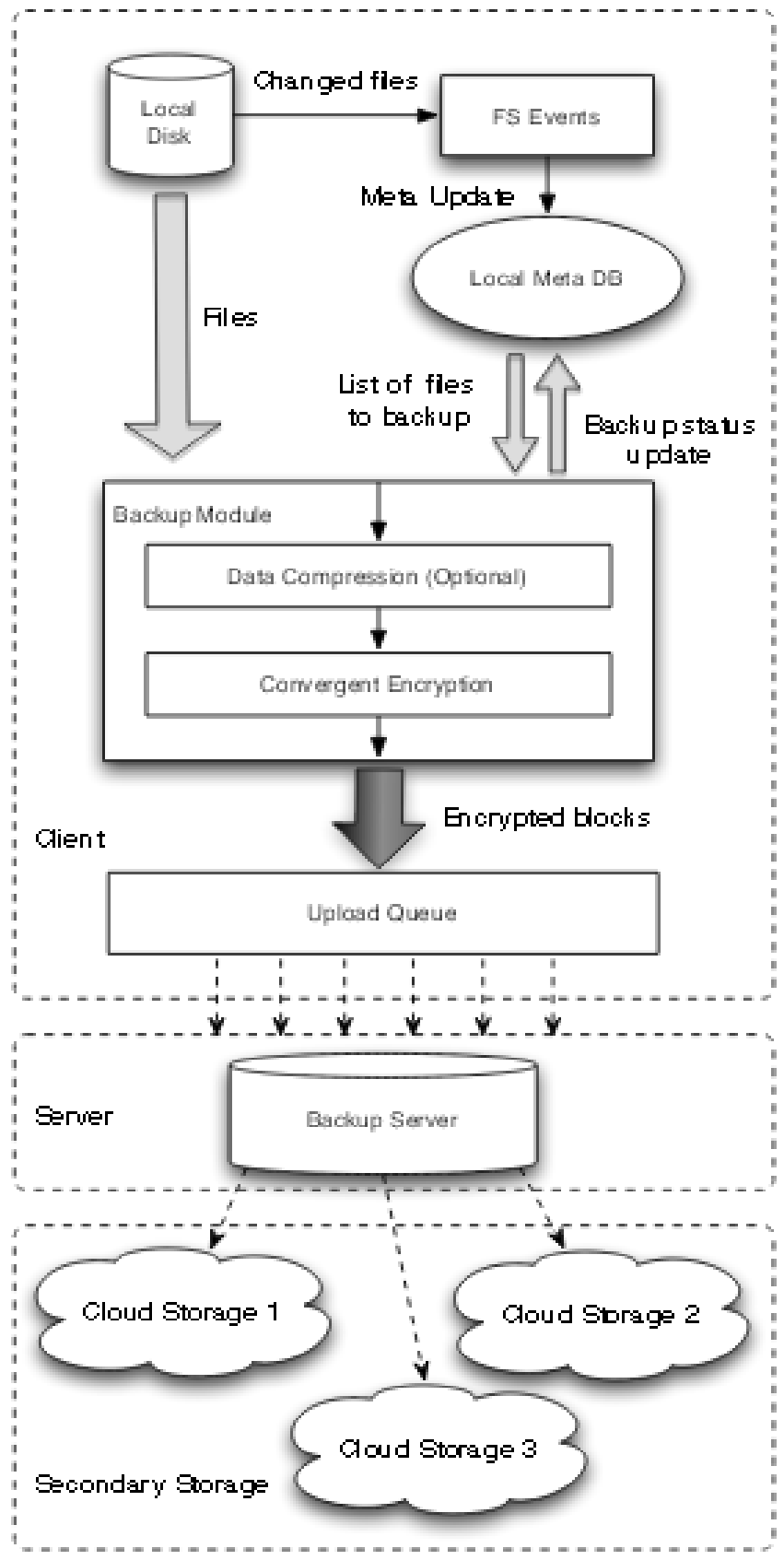

Figure 3: System diagram

Different types or individual data now store extensive amounts of individual and corporate information on tablets or home PCs.

By doing this type of work it is helpless against burglary or equipment disappointment. Ordinary ideal arrangements are not appropriate to this environment, and reinforcement administrations are every now and again deficient. This paper depicts a calculation which exploits the information which is basic between clients to build the pace of reinforcements, and diminish the capacity necessities. This calculation bolsters customer end per-client encryption which is essential for classified individual information. It likewise underpins a one of a kind element which permits prompt location of normal sub trees, dodging the need to question the reinforcement framework for each document. It means the same data uses by different users have take large space and reduce the performance of your PC. We portray a model usage of this 


\section{International Journal of Science and Research (IJSR) \\ ISSN (Online): 2319-7064}

Index Copernicus Value (2015): 78.96 | Impact Factor (2015): 6.391

calculation for Apple OS X, and present an investigation of the potential viability, utilizing genuine information acquired from an arrangement of ordinary clients. At last, we talk about the utilization of this model in conjunction with remote distributed storage, and present an investigation of the commonplace cost reserve funds [2].

\section{Secure Deduplication with Efficient and Reliable Convergent Key Management}

Deduplication is a system for taking out copy duplicates of information, and has been broadly utilized as a part of distributed storage to decrease storage space and transfer data transfer capacity. Promising as it may be, an emerging test is to perform secure deduplication in distributed storage.

Albeit joined encryption has been widely received for secure deduplication, a basic issue of making focalized encryption down to earth is to productively and dependably deal with an immense number of united keys. This paper makes the first endeavor to formally notify the issue of accomplishing effective and dependable key administration in secure deduplication. Firstly we introduce a pattern approach in which every client holds an autonomous expert key for scrambling the aim keys and outsourcing them to the cloud.

On the second way, such a standard key administration plan produces a tremendous number of keys with the expanding number of clients and obliges clients to dedicatedly secure the expert keys. To this end, we propose Dekey, another development in which clients don't have to deal with any keys all alone however rather safely circulate the united key shares over different servers. Security examination exhibits that Dekey is secure as far as the definitions determined in the proposed security model. As a proof of idea, we actualize Dekey utilizing the Ramp mystery sharing plan and show that Dekey brings about restricted overhead in reasonable situations [3].

\section{Proofs of Ownership in Remote Storage Systems}

Distributed storage frameworks are turning out to be progressively prominent. A promising innovation that holds their expense down is deduplication, which stores just a solitary duplicate of rehashing information. Customer side deduplication endeavors to recognize deduplication opportunities as of now at the customer and save the transmission capacity of transferring duplicates of existing documents to the server. After that process we looks assaults that endeavor customer side deduplication, permitting an aggressor to access self-assertive size records of different clients in view of a little hash marks of these documents.

All the more particularly, an aggressor who knows the hash mark of a record can persuade the capacity benefit that it possesses that document, henceforth the server lets the assailant download the whole record. (In parallel to our work, a subset of these assaults were as of late presented in the wild regarding the Dropbox record synchronization administration). To overcome of this problem, we present the thought of verifications of-possession (PoWs), which lets a customer effectively present to a server that that the customer holds a document, as opposed to simply some short data about it. We formalize the idea of evidence of-proprietorship, under thorough security definitions, and thorough productivity prerequisites of Petabyte scale stockpiling frameworks. We then present arrangements in view of Merkle trees and particular encodings, and investigate their security. We actualized one variation of the plan. Our execution estimations show that the plan causes just a little overhead contrasted with guileless customer side deduplication [4]

\section{E. RevDedup: A Reverse Deduplication Storage System Optimized for Reads to Latest Backups}

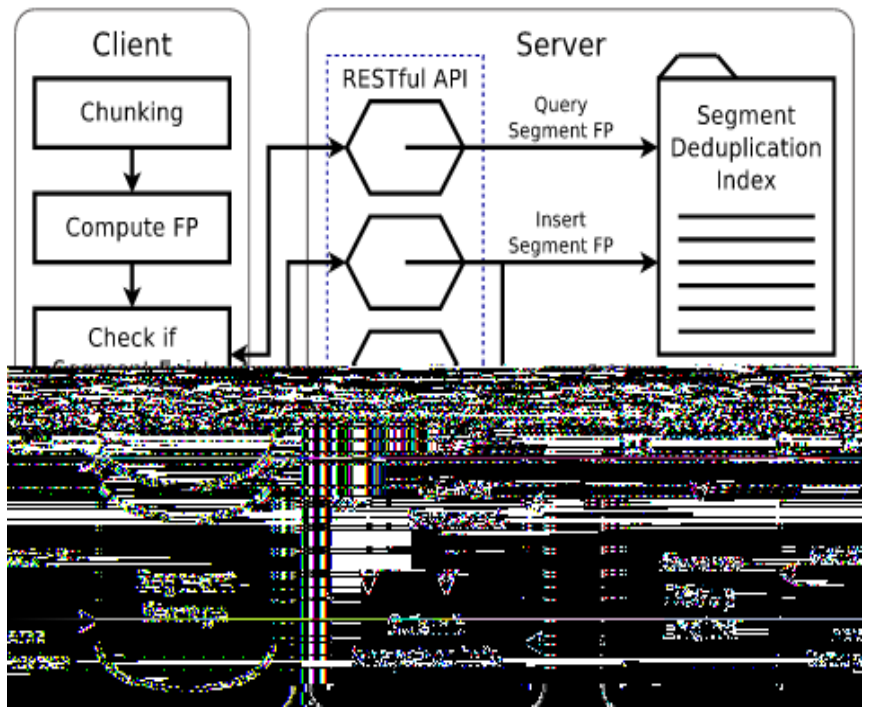

Figure 4: Reverse duplication

Scaling up the reinforcement stockpiling for a perpetually expanding volume of virtual machine (VM) pictures is a basic issue in virtualization situations. While deduplication is known not dispose of copies for VM picture capacity, it additionally presents fracture that will corrupt read execution. We propose RevDedup, a deduplication framework that upgrades peruses to most recent VM picture reinforcements utilizing a thought called reverse deduplication. Conversely with traditional deduplication that describe copies from new information, RevDedup describe copies from old information, in this way moving odd to old information while keeping the design of new information as consecutive as would be prudent. We assess our RevDedup model utilizing miniaturized scale benchmark and certifiable workloads. For a 12-week compass of certifiable VM pictures from 160 use rs, RevDedup accomplishes high deduplication productivity with around $97 \%$ of sparing, and high reinforcement and read throughput on the request of $1 \mathrm{~GB} / \mathrm{s}$. RevDedup additionally brings about little metadata overhead in reinforcement/read operations [5].

\section{Volume 6 Issue 1, January 2017




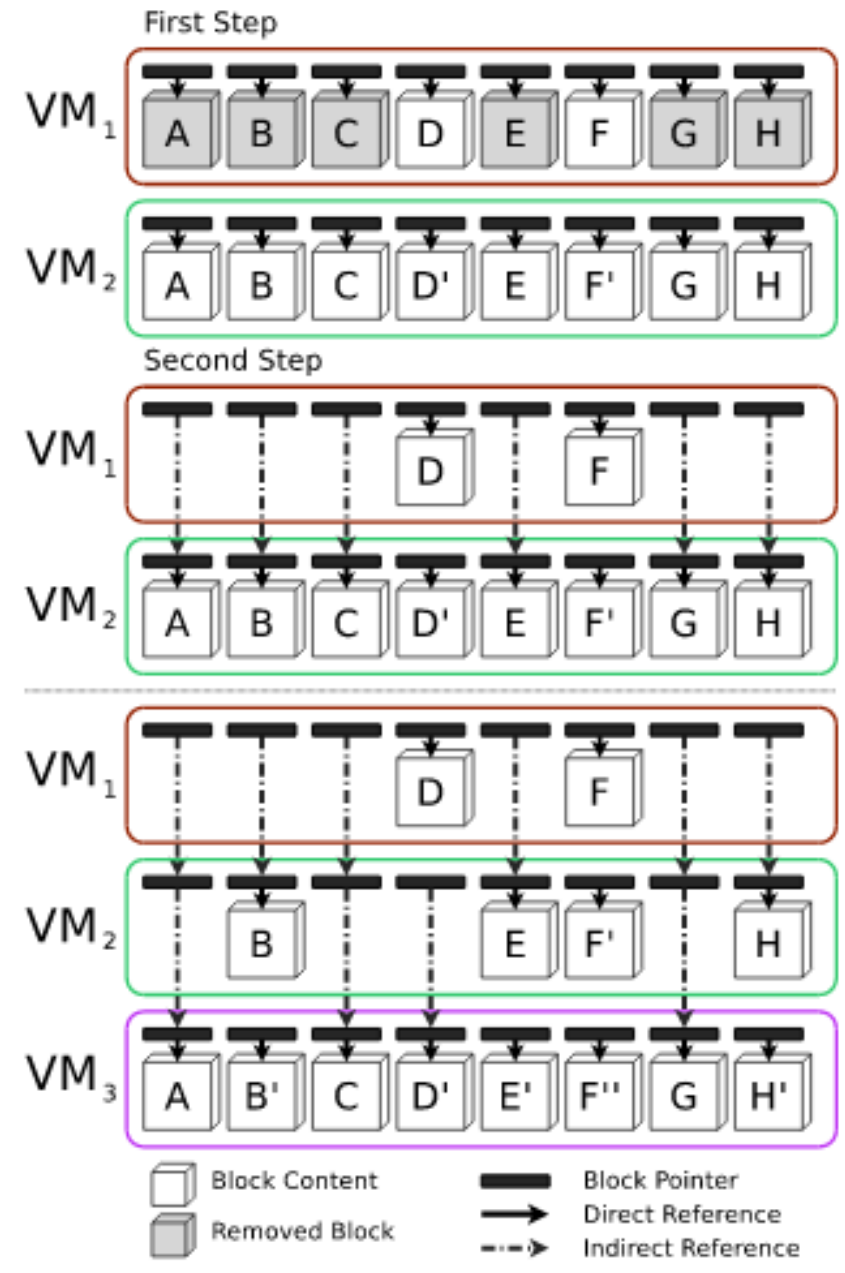

Figure 5: Reverse duplication example

F. Private Data Deduplication Protocols in Cloud Storage Another idea namely call private information deduplication convention, a deduplication system for private information stockpiling is presented and formalized. Naturally, a private information deduplication convention allow a customer who holds a private information demonstrates to a server who have a synopsis string of the information that he/she is the proprietor of that information without uncovering additional data to the server. Our idea can be seen as a supplement of the cutting edge open information deduplication conventions of Halevi et al. The security of private information deduplication conventions is formalized in the recreation based system in the connection of two-gathering calculations. A development of private deduplication conventions in view of the standard cryptographic suspicions is then introduced and examined. We demonstrate that the proposed private information deduplication convention is provably secure accepting that the basic hash capacity is crash flexible, the discrete logarithm is hard and the eradication coding calculation can deletion up to $\alpha$-division of the bits in the vicinity of malignant enemies in the vicinity of vindictive foes.

To the best our insight this is the first deduplication convention for private information stockpiling [6].

\section{Conclusion}

Here we provided reason that our proposed framework information DE duplication of record is done approves way and safely. In this we have additionally proposed new duplication check system which produce the token for the private document. The information client needs to present the benefit alongside the united key as a proof of possession. We have settled more basic piece of the cloud information stockpiling which is just endured by diverse systems. Proposed routines guarantee the information duplication safely.

\section{References}

[1] M. Bellare, S. Keelveedhi, and T. Ristenpart. Dupless: Serveraided encryption for deduplicated storage. In USENIX Security Symposium, 2013.

[2] P. Anderson and L. Zhang. Fast and secure laptop backups with encrypted de-duplication. In Proc. of USENIX LISA, 2010.

[3] J. Li, X. Chen, M. Li, J. Li, P. Lee, andW. Lou. Secure deduplication with efficient and reliable convergent key management. In IEEE Transactions on Parallel and Distributed Systems, 2013.

[4] S. Halevi, D. Harnik, B. Pinkas, and A. Shulman-Peleg. Proofs of ownership in remote storage systems. In Y. Chen, G. Danezis, and V. Shmatikov, editors, ACM Conference on Computer and Communications Security, pages 491-500. ACM, 2011.

[5] C. $\mathrm{Ng}$ and P. Lee. Revdedup: A reverse deduplication storage system optimized for reads to latest backups. In Proc. of APSYS, Apr 2013.

[6] W. K. Ng, Y. Wen, and H. Zhu. Private data deduplication protocols in cloud storage. In S. Ossowski and P. Lecca, editors, Proceedings of the 27th Annual ACM Symposium on Applied Computing, pages 441446. ACM, 2012. 